\title{
Zinc-induced envelope stress diminishes type III secretion in enteropathogenic Escherichia coli
}

\author{
Jay L Mellies ${ }^{1 *}$, Katherine Thomas ${ }^{1}$, Michael Turvey ${ }^{1}$, Neil R Evans ${ }^{1}$, John Crane ${ }^{2}$, Ed Boedeker ${ }^{3}$ \\ and Gregory C Benison ${ }^{1}$
}

\begin{abstract}
Background: Dietary supplementation with zinc has been shown to reduce the duration and severity of diarrhoeal disease caused by Enteropathogenic Escherichia coli, common in infants in developing countries. Initially this therapeutic benefit was attributed to the correction of zinc deficiency in malnourished individuals, but recently evidence has emerged that zinc significantly impacts the pathogens themselves: zinc concentrations achievable by oral supplementation can reduce the expression of key virulence-related genes in EPEC and related organisms.

Results: Here, we investigate three possible mechanisms for such zinc-induced changes in expression of EPEC virulence: direct interaction of zinc with regulators of LEE operons; genetic interaction of LEE operons with known regulators of zinc homeostasis; and finally, downregulation of LEE transcription associated with activation of the $\sigma^{\mathrm{E}}$ envelope stress response by zinc. We find evidence only for the latter mechanism, including zinc-induced down-regulation of type III secretion in EPEC similar to that caused by ammonium metavanadate, another known inducer of the $\sigma^{\mathrm{E}}$ stress response.
\end{abstract}

Conclusions: We conclude therefore that envelope stress is a major mechanism by which zinc attenuates the virulence of EPEC and related pathogens.

\section{Background}

Enteropathogenic Escherichia coli (EPEC) is a leading cause of infantile diarrhoea in developing countries [1]. Net secretory diarrhoea results from altered host cell signaling events, loosening of tight junctions and is exacerbated by the destruction of absorptive tissue, the host intestinal microvilli [2]. These phenotypes are mediated by a type III secretion system, a molecular syringe that secretes bacterial proteins into host cells, and is a common feature of many gram-negative pathogens [3]. The mechanism of EPEC diarrhoeal disease is similar to that of enterohemorrhagic E. coli (EHEC), and thus EPEC can be used as a surrogate for investigating disease caused by this more serious threat to public health. While, by definition, EPEC, possesses no diffusible toxins, EHEC in contrast

*Correspondence: jay.mellies@reed.edu

1 Biology Department, Reed College, Portland OR, USA

Full list of author information is available at the end of the article produces Shiga toxin, causing bloody diarrhoea or hemorrhagic colitis. The production of Shiga toxin also can lead to the life-threatening complication, hemolytic uremic syndrome (HUS), which occurs in approximately $10 \%$ of reported cases of EHEC infection [4].

In developing countries, studies have shown that administering zinc to children with diarrhoea reduces the severity of disease $[5,6]$. It was initially hypothesized that this effect was due to correction of zinc deficiencies often seen in impoverished and malnourished children in these regions of the world. Certainly zinc is an important nutrient due to its fundamental role as a cofactor - over 300 zinc-depedent enzymes have been identified from all forms of life, with many of these such as carbonic anhydrase forming a basic part of human metabolism. Zinc is also found in non-enzymatic contexts in humans, for example its structural role in the ubiquitous zinc finger transcriptional regulators [7]. Zinc is also important for immune function, and zinc deficiency adversely affects the health and development of children $[8,9]$. However, a 
double-blind, randomized, controlled study involving 937 children with acute diarrhoea conducted in New Delhi, India demonstrated that zinc supplementation benefited children in the experimental group irrespective of the child's initial plasma zinc level [5]. Thus beyond being an important co-factor necessary for immune and enzyme function in children, zinc also reduces the duration and severity of diarrhoeal disease caused by E. coli. For an initial study conducted in Calcutta, many, but not all of the reported cases were caused by EPEC. Thus some researchers have argued for greater use of zinc supplementation to treat bacterial diarrhoeal disease in children in the developing world [10].

In EPEC, zinc causes a reduction in net protein secretion via the type III secretion system [11], encoded within the pathogenicity island termed the locus of enterocyte effacement or LEE. As an underlying mechanism, this metal ion reduces the expression of espA, encoding the monomer of the molecular syringe, and $\operatorname{esp} B$, which in conjunction with $\operatorname{espD}$ encodes the pore that inserts into the membrane of the host cell [12]. The genes $\operatorname{esp} A, \operatorname{esp} B$ and $\operatorname{espD}$ are found within the LEE4 operon of EPEC $[13,14]$. Evidence suggests that zinc dependent down regulation of LEE4 involves the global regulator protein Ler, encoded within the $L E E 1$ operon. Zinc also reduces expression of $L E E 1$, and thus Ler [11].

In our current study we sought to understand the underlying mechanism of how zinc reduces the expression of LEE genes of EPEC. We found no evidence to suggest that zinc directly acts on the regulatory protein Ler. Rather, we present evidence that zinc causes EPEC envelope stress, leading to a $\sigma^{\mathrm{E}}$-dependent stress response characterized by increased expression of rpoE. Treating EPEC with ammonium metavanadate $\left(\mathrm{NH}_{4} \mathrm{VO}_{3}\right)$ - a known chemical inducer of the $\sigma^{\mathrm{E}}$-dependent response - caused a reduction in type III-dependent secretion similar to that observed in the presence of zinc. This is a first account of a specific mechanism on how zinc supplements reduce the duration and severity of disease caused by EPEC and related diarrhoeal pathogens.

\section{Results}

Millimolar concentrations of zinc are required to inhibit Ler binding

Previous studies indicated that exogenous zinc diminished EPEC pathogenesis, in part, by inhibiting expression of virulence genes. Specifically, expression of genes of the LEE, encoding components of the type III secretion system, were reduced in the presence of 0.1 to $0.5 \mathrm{mM}$ zinc acetate $[11,15]$. Data suggested that, for the LEE4 operon, encoding $\operatorname{esp} A$, zinc-dependent down-regulation required the global regulator Ler [14], which controls expression of the LEE4 operon. Thus we initially posited that upon zinc stress cytoplasmic concentrations of this metal ion prevented Ler binding to LEE4 regulatory DNA.

To test this hypothesis, we performed electrophoretic mobility shift assays (EMSA) using purified components (Figure 1). One hundred nanograms of LEE4 regulatory DNA was incubated with $500 \mathrm{nM}$ Ler protein with increasing amounts of zinc acetate. In the absence of added zinc, the Ler/DNA complex migrated poorly into the polyacrylamide gel compared to the DNA fragment alone, consistent with previously published data $[16,17]$. Concentrations of added zinc acetate up to $100 \mu \mathrm{M}$ showed no effect on the ability of Ler protein to bind and shift the $L E E 4$ regulatory DNA (Figure 1). At $1000 \mu \mathrm{M}$, or $1 \mathrm{mM}$, zinc acetate we observed reduction in the ability of Ler to bind LEE4 DNA by $80 \%$. Thus in vitro, millimolar concentrations of zinc were necessary to disrupt Ler binding to regulatory DNA sequences.

Under normal physiological conditions, it is estimated that the concentration of free zinc within $E$. coli is in the femtomolar range, less then one zinc atom per cell [18], whereas the zinc quotient of the cell- that complexed with amino acids, ribosomal proteins and enzymesreaches micromolar concentrations. Because millimolar

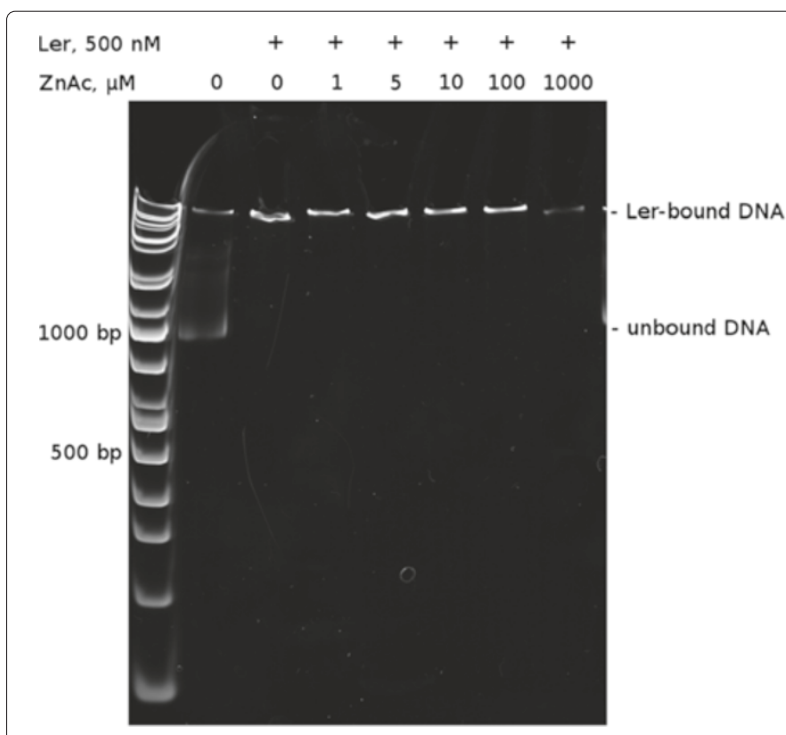

Figure 1 Sub-millimolar zinc does not interfere with Ler binding to the LEE4 operon in vitro. Ler binding to a fragment containing the LEE4 promoter (bases -468 to +460 relative to the transcription start point) was assessed by EMSA in the presence of varied zinc acetate concentrations. Purified Ler protein at a final concentration of $0.5 \mu \mathrm{M}$ was incubated with $100 \mathrm{ng}$ DNA at room temperature for $15 \mathrm{~min}$, then separated on a non-denaturing $5 \%$ polyacrylamide gel by electrophoresis at $40 \mathrm{~V}$ for 16 hours at $4^{\circ} \mathrm{C}$. When Ler was present, essentially all of the DNA was bound in a nucleoprotein complex which was not disrupted by zinc acetate at any concentration up to $100 \mu \mathrm{M}$, and only partially at $1000 \mu \mathrm{M}$ (the highest concentration tested). The upper and lower arrows mark the locations of bound and unbound DNA, respectively. 
concentrations of zinc acetate were necessary for disrupting Ler binding to LEE4 (Figure 1) and no putative zinc binding domains are found within Ler (data not shown), we concluded that alterations of LEE gene expression by zinc did not involve direct interaction of zinc with the regulatory protein Ler.

\section{LEE gene expression is reduced by zinc in K-12 laboratory strains}

To further our understanding of zinc alteration of LEE gene expression we transformed plasmids containing LEE1-lacZ and LEE4-lacZ fusions (pJLM164 and pJLM165; Table 1) into the prototypical EPEC strain E2348/69, EPEC strain LRT9, strain JPN15 lacking the EAF virulence plasmid, and the K-12 strain MC4100. Strains were grown in DMEM medium in the presence and absence of $0.5 \mathrm{mM}$ zinc acetate, and assayed for $\beta$-galactosidase activity. $\beta$-galactosidase activity derived from the LEE4 operon was significantly diminished in the presence of zinc in all four strains (Figures 2A-D). Similarly, $\beta$-galactosidase activity derived from the LEE1lac $Z$, multi-copy fusion was also diminished by the presence of $0.5 \mathrm{mM}$ zinc acetate in the four strains tested (Figures 2E-H).

As a control we determined whether $0.5 \mathrm{mM}$ zinc acetate affected the growth rate of either EPEC or the laboratory strain MC4100. We found that the doubling times of EPEC strain E2348/69 were 93 and 104 minutes in DMEM for 0 or $0.5 \mathrm{mM}$ zinc acetate added, whereas for MC4100 the doubling times were 41 and 77 minutes for 0 and $0.5 \mathrm{mM}$ zinc acetate, respectively. Thus the growth rate of the pathogenic strain E2348/69 was slowed by $\sim 10 \%$ though that of the laboratory strain was more adversely affected by zinc. These results indicated that previous assays demonstrating zinc-mediated downregulation of LEE genes using qRT-PCR $[11,15]$ could be faithfully reproduced using a lac $Z$ reporter gene system, that down-regulation of LEE4 occurred in the absence of Ler in the K-12-derived strain MC4100, and because we could observe this regulation in MC4100 derivatives that the regulation was not specific to the EPEC pathotype.

\section{Down-regulation of LEE genes by zinc occurs in the} absence of zinc ion homeostasis regulators Zur and ZntR

We took advantage of the fact that zinc down-regulation of LEE genes could be reconstituted in K-12-derived strains to determine whether the observed regulation involved regulators of zinc ion homeostasis. The Zur regulator represses expression of the $z n u A B C$ zinc transporter when the bacterium has excess intracellular concentrations of zinc, while ZntR stimulates expression of the $z n t A$ exporter when excess concentrations of zinc are found within the cytoplasm [18,29]. In the MC4100 $\triangle z$ ur strain SIP812 containing the pJLM164 plasmid, $\beta$-galactosidase activity derived from the LEE1 operon decreased from $\sim 5000$ to 1000 Miller units in the presence of $0.3 \mathrm{mM}$ zinc acetate, a 5 -fold reduction (Student's $\mathrm{t}$-test; $\mathrm{n}=3 ; \mathrm{p}<0.05)$. Similarly, in the MC4100 $\Delta z h t R$ strain containing the pJLM164 plasmid $\beta$-galactosidase activity decreased from $\sim 3500$ to 500 Miller units, a 7-fold

Table 1 Bacterial strains and plasmids used in this study

\begin{tabular}{|c|c|c|}
\hline Strain or plasmid & Genotype or description & Source or reference \\
\hline \multicolumn{3}{|l|}{ Strains } \\
\hline E2348/69 & Prototype EPEC strain (serotype 0127:H6) & {$[19]$} \\
\hline JPN15 & EAF plasmid-cured derivative of E2348/69 & {$[20]$} \\
\hline MC4100 & $\operatorname{araD139\Delta } \Delta(\operatorname{argF}-$ lac $)$ U169 rpsL150 relA1 flbB5301 deoC1 ptsF25 rbsR & {$[21]$} \\
\hline JLM164 & MC4100 ФLEE $1-l a c Z$ & {$[14]$} \\
\hline$J L M 165$ & MC4100 ФLEE4 - lacZ & {$[14]$} \\
\hline SIP812 & MC4100 zur::Spc ${ }^{r} /$ Str $^{r}$ & {$[22]$} \\
\hline TB742 & MC4100 $\Delta z n t R$ & {$[23]$} \\
\hline СТ32 & MC4100 rpoE - lacZ & {$[24]$} \\
\hline MCamp & MC4100 bla - lacz & {$[25]$} \\
\hline CVD452 & E2348/69 $\Delta$ escN :: aphT & {$[26]$} \\
\hline LRT-9 & EPEC O111:abH2 & {$[27]$} \\
\hline \multicolumn{3}{|l|}{ Plasmids } \\
\hline pRS551 & Promoterless lacZ reporter fusion vector & {$[28]$} \\
\hline pVSAPR & $b l a-l a c z$ & {$[25]$} \\
\hline pJLM164 & LEE $1-\operatorname{lac} Z$ & {$[14]$} \\
\hline pJLM165 & LEE4 - lacZ & [14] \\
\hline
\end{tabular}




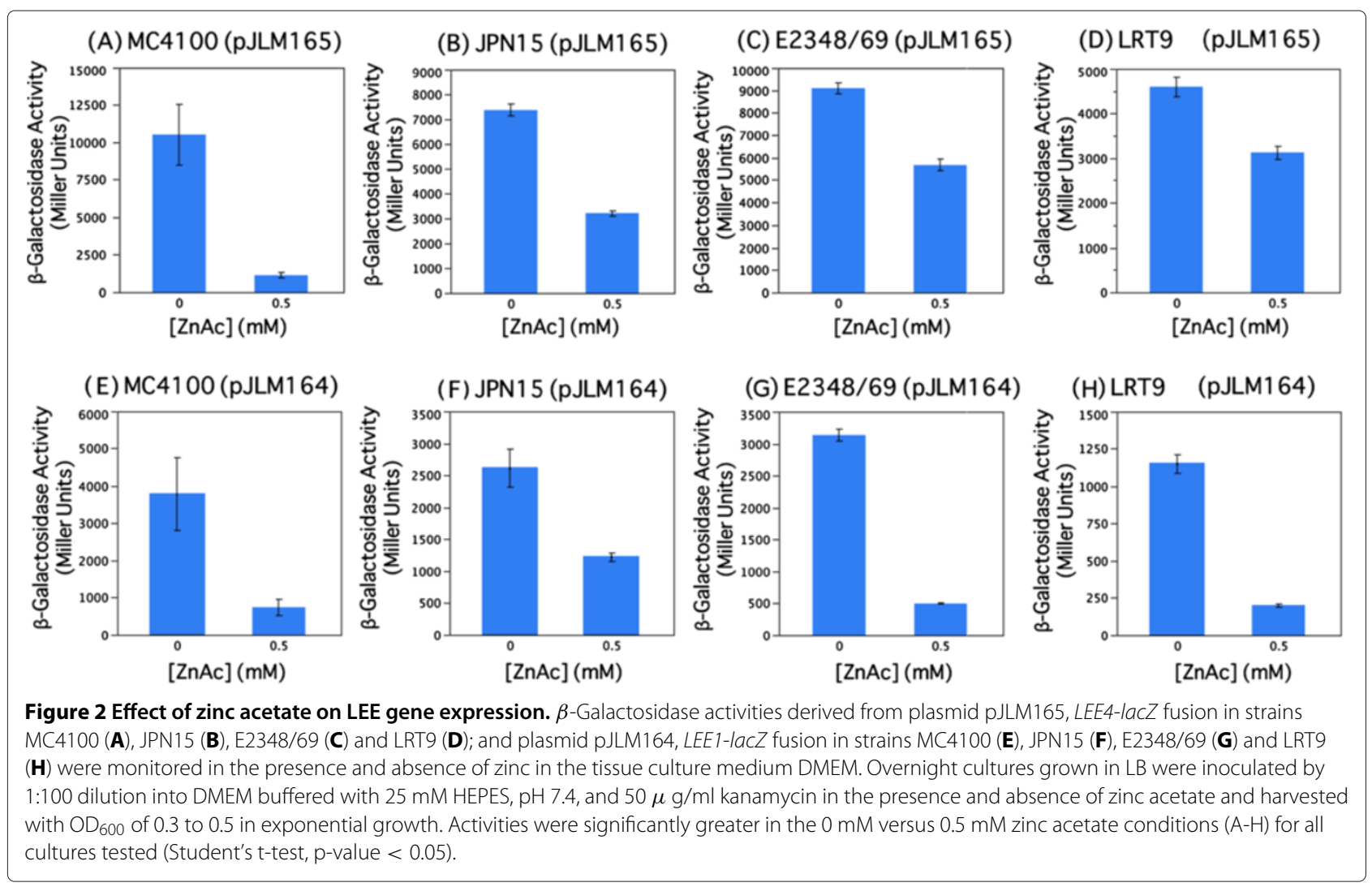

reduction (Student's t-test; $\mathrm{n}=3 ; \mathrm{p}<0.05$ ), in the presence of $0.3 \mathrm{mM}$ zinc acetate. We therefore concluded that zinc-mediated repression of $L E E 1$, encoding Ler, did not require the global regulators of zinc homeostasis Zur or ZntR.

\section{Zinc stress increases rpoE expression}

Previous publications have indicated that excess zinc induces the expression of genes involved in envelope stress $[30,31]$. While these studies were conducted in complex media, including LB, we wanted to determine if genes associated with envelope stress were stimulated by zinc in the tissue culture medium DMEM used in our assays. We therefore plated the MC4100-derived strains CT32, containing a single-copy $r p o E-l a c Z$ fusion, JLM164 and JLM165, containing LEE1-lacZ and LEE4-lacZ fusions, respectively, and as a negative control, strain MCamp containing a single-copy bla-lacZ fusion on DMEM agar. Sterile disks containing $15 \mu \mathrm{l}$ of varying concentrations of zinc acetate were placed on the lawns of bacteria on selective medium containing $\mathrm{X}$-gal, and growth proceeded overnight at $37^{\circ} \mathrm{C}$.

A relatively small zone of growth inhibition was noted surrounding the disk containing $100 \mathrm{mM}$ zinc acetate for all strains tested (Figure 3). Thus high concentrations of zinc inhibited growth of these MC4100 derivatives.
Consistent with our previous assays, we observed decreased $\beta$-galactosidase activities, indicated by a lack of blue color, surrounding the zinc acetate-containing disks on the plates containing the JLM164 and JLM165 strains, demonstrating that LEE1 and LEE4 expression was down-regulated in the presence of zinc acetate. However, we also observed similar down-regulation of $\beta$ galactosidase activity derived from the bla-lac $Z$ negative control fusion from strain MCamp, suggesting that zinc caused a generalized down-regulation of gene expression in E. coli.

In contrast to these results, we did not observe a downregulation of the rpoE-lacZ fusion from strain CT32 in the presence of any of the zinc acetate concentrations tested, indicated by blue color directly adjacent to the disks (Figure 3D). Consistent with this observation, by Miller assay [32], $\beta$-galactosidase activity derived from the rpoE-lacZ fusion strain CT32 in DMEM increased 1.7fold from $512 \pm 24$ to $865 \pm 19$ Miller units (Student's $\mathrm{t}$-test; $\mathrm{n}=3 ; \mathrm{p}<0.05)$ in the presence of $0.3 \mathrm{mM}$ zinc acetate. Because rpoE expression occurs via a mechanism whereby the alternate sigma factor $r p o E$ is released from the cytoplasmic membrane upon insult [33], we concluded that E. coli grown in DMEM experiences envelope stress in the presence of zinc acetate, consistent with previously published reports using complex media [30,31]. 

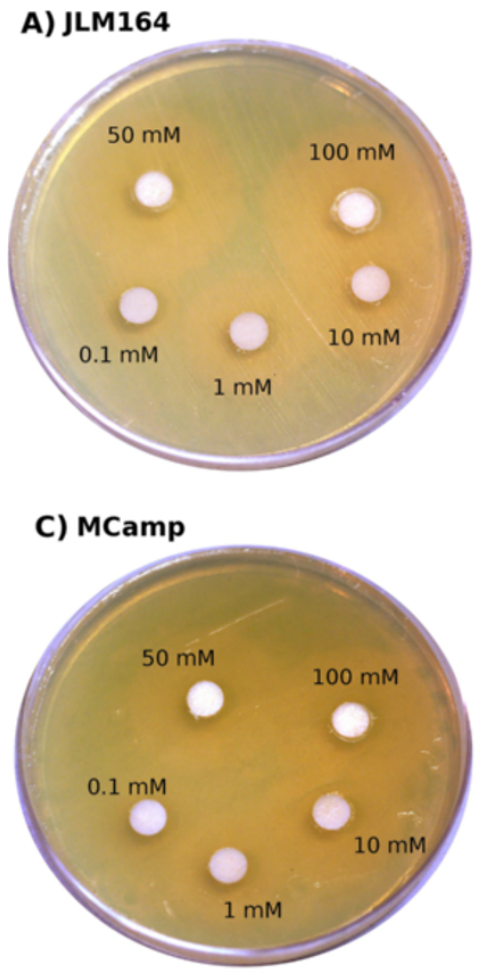

B) JLM165

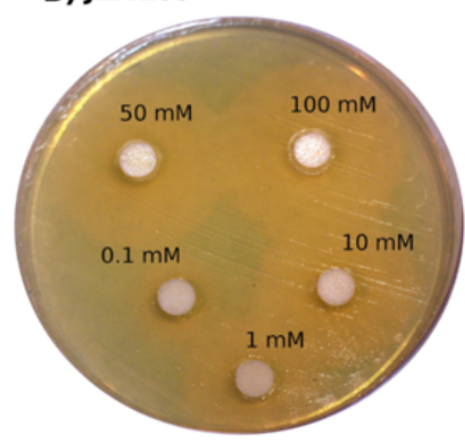

D) $\mathrm{CT} 32$

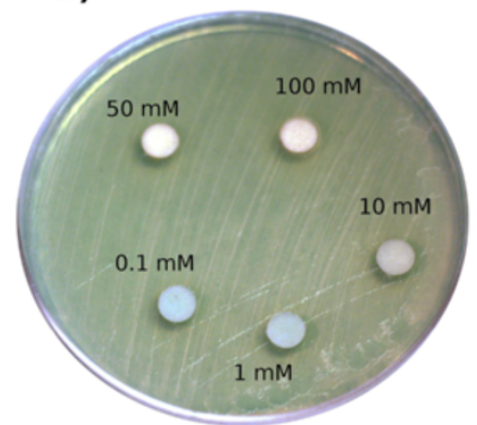

Figure 3 Zinc downregulates both genes related and non-related to virulence but not $\mathbf{r p o E}$. Overnight cultures of single-copy lac $Z$ fusions JLM164 (LEE1 - lacZ; A), JLM165 (LEE4 - lacZ; B), MCamp (bla - lacZ; C), and CT32 (rpoE - lacZ; D) were spread evenly onto DMEM plates containing $30 \mathrm{mg} / \mathrm{ml} \mathrm{X-gal.} \mathrm{Discs} \mathrm{of} \mathrm{sterile} \mathrm{filter} \mathrm{paper} \mathrm{were} \mathrm{dropped} \mathrm{onto} \mathrm{the} \mathrm{lawn;} 15 \mu \mathrm{l}$ of different concentrations of zinc acetate were placed on each disc $(100 \mathrm{mM}, 50 \mathrm{mM}, 10 \mathrm{mM}, 1 \mathrm{mM}, 0.1 \mathrm{mM})$. These plates were grown for approximately 18 hours and then moved to $4^{\circ} \mathrm{C}$ for 6 hours to develop the blue color. Virulence genes were downregulated in the presence of zinc (A \& B), but so was the bla gene encoding $\beta$-lactamase (C). In contrast, rpoE was not downregulated in the presence of zinc (D). Also of note is the small $(\sim 1 \mathrm{~mm})$ zone of growth inhibition around the $100 \mathrm{mM}$ and $50 \mathrm{mM}$ discs.

\section{Zinc damages the EPEC envelope}

To substantiate the damage to the EPEC envelope, comprising the inner membrane, periplasmic space, and outer membrane, suggested by our reporter gene fusion assays, we performed electron microscopy. EPEC bacteria were grown in DMEM tissue culture medium in the absence and presence of $0.3 \mathrm{mM}$ zinc acetate. In the absence of zinc, the envelope of the bacteria appeared intact (Figures 4A-C). However, after growth in DMEM in the presence of zinc the outer membrane of the bacteria appeared compromised, and we observed what appeared to be multiple membrane blebs on individual bacteria (Figures 4D,E). Furthermore, we also observed bacteria with irregularly shaped inner membranes (Figure 4F). These data provided direct evidence that zinc damages the EPEC envelope.

\section{Chemical disruption of the EPEC envelope diminishes type III secretion}

Zinc stimulates the expression of rpoE (Figure 3) and physically damages the EPEC envelope (Figure 4). These data demonstrated that, as for laboratory strains of E. coli, zinc causes envelope stress in EPEC. Along with downregulation of LEE genes encoding type III secretion system components envelope stress could, at least in part, explain why zinc reduces diarrhoea in a rabbit illeal loop model of infection [11]. To test this hypothesis we monitored proteins secreted from EPEC grown in DMEM in the presence of ammonium metavanadate $\left(\mathrm{NH}_{4} \mathrm{VO}_{3}\right)$. Ammonium metavanadate causes envelope stress and specifically stimulates the rpoE regulon $[24,34]$. Thus our prediction was that this chemical, in a manner similar to zinc, would diminish protein secretion via the type III secretion system of EPEC strain E2348/69.

To test this prediction strain E2348/69 was grown in DMEM overnight, in static cultures in the presence of increasing concentrations of $\mathrm{NH}_{4} \mathrm{VO}_{3}$. Bacteria were pelleted, and secreted proteins were harvested from the supernatant by TCA-precipitation. To control for proteins being released from the bacteria independently from the type III secretion system, we also harvested supernatant proteins from the strain CVD452, deleted for escN, encoding the ATPase [26]. We monitored secretion in the presence of zinc because protein secretion was previously 


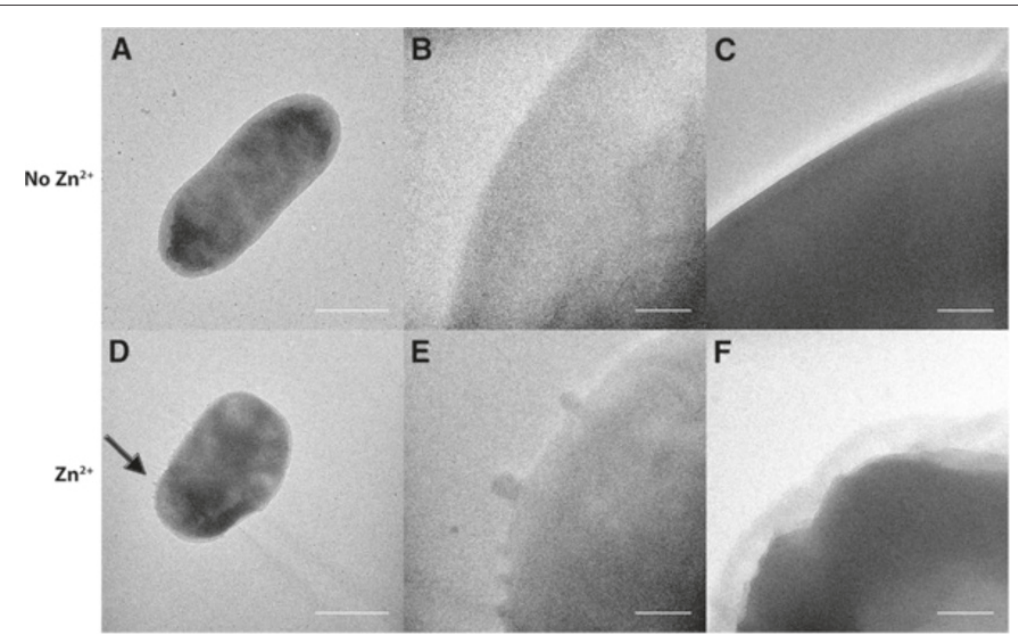

Figure 4 The effects of zinc stress on the EPEC envelope imaged by transmission electron microscopy. After 10-hour growth in DMEM medium, cultures were grown for an additional 5 hours in the absence $\mathbf{( A , B )}$ and presence $\mathbf{( D , E )}$ of $0.3 \mathrm{mM}$ zinc acetate. EPEC bacteria were pelleted, the medium discarded, and bacteria then were resuspended in $0.1 \mathrm{M} \mathrm{MgSO}_{4}$. Samples were placed on carbon formvar grids, stained with $1.3 \%$ uranyl acetate and viewed by transmission electron microscopy. The same procedure was repeated with 1-hour growth in DMEM medium, followed by an additional 5-hours of growth in the absence (C) and presence (F) of $0.3 \mathrm{mM}$ zinc acetate. Arrow points to outer membrane blebs in (D). (A,D) Bars $1.0 \mu \mathrm{m}$; (B-C,D-F) Bars $0.1 \mu \mathrm{m}$.

shown to be diminished in the presence of this metal ion [11].

Upon separation by SDS-PAGE, for wild type strain E2348/69 we observed the EspB protein migrating to $~ 33$ $\mathrm{kDa}$, which was dependent upon the type III system for secretion given that this band was absent in the lane corresponding to the $\Delta e s c N$ strain CVD452 (Figure 5; [35]). The identity of EspB was confirmed by an in-gel tryptic digest followed by mass spectrometry (data not shown). Increasing concentrations of $\mathrm{NH}_{4} \mathrm{VO}_{3}$ caused diminished protein secretion in a concentration dependent manner, such that at $10 \mathrm{mM}$ of this chemical secretion of EspB was diminished by more than $70 \%$. Because $\mathrm{NH}_{4} \mathrm{VO}_{3}$ stresses the bacterial envelope, specifically targeting the RpoE stress pathway, we concluded that stress to the EPEC envelope caused decreased protein secretion via the type III secretion system.

\section{Zinc precipitates phosphate from the tissue culture medium DMEM}

Through the course of growing EPEC cultures in DMEM we observed that, not the doubling time, but rather the growth yield was modestly diminished in the presence of zinc acetate (data not shown). In addition, $\mathrm{CFU} / \mathrm{ml}$ values after overnight growth in DMEM were $\sim 1.0 \times 10^{9}$ versus $5.0 \times 10^{8}$ in the absence and presence of $0.3 \mathrm{mM}$ zinc. As

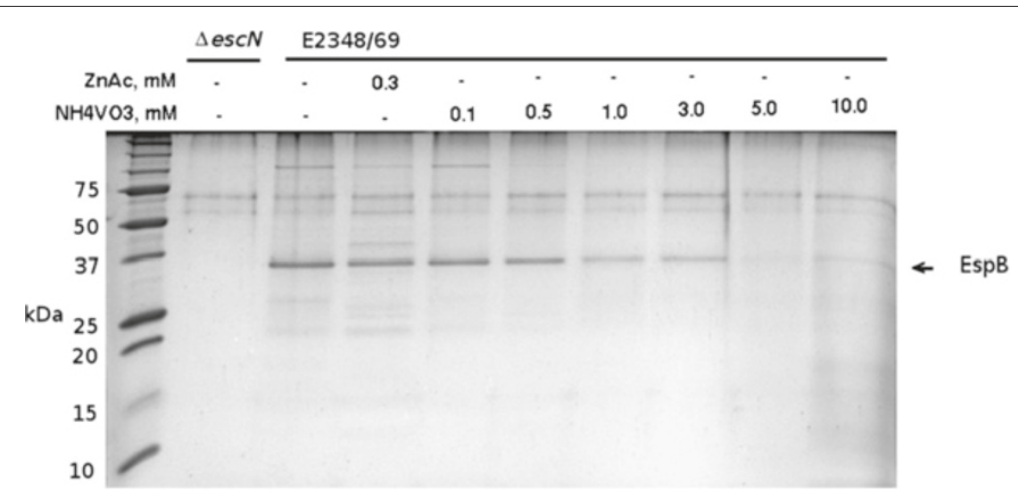

Figure 5 Zinc and ammonium metavanadate both inhibit protein secretion from EPEC. Cultures of EPEC strain E2348/69 were grown statically overnight in DMEM with varied concentrations of zinc acetate or ammonium metavanadate to an $\mathrm{OD}_{600}$ of 0.8 - 1.0. A culture of an EPEC strain deficient in type III secretion ( $\Delta e s c N$ ) was included as a control. Cells were removed by centrifugation, then proteins in the culture medium were precipitated with $25 \%$ trichloroacetic acid and visualized with SDS-PAGE. The volume of supernatant precipitated was chosen such that volume $(\mathrm{ml}) \times$ culture $\mathrm{OD}_{600}=6.0$. 
phosphate is present in DMEM at $1 \mathrm{mM}$ concentration, zinc phosphate is insoluble in solution, and we observed a small amount of white precipitate in DMEM in the presence of zinc acetate (data not shown), we hypothesized that the addition of zinc removed phosphate from this tissue culture medium. Indeed we observed that after the addition of millimolar concentrations of zinc, the concentration of soluble phosphate diminished in a linear fashion in DMEM (Figure 6). Therefore we concluded that zinc removed the essential element phosphorous from solution, and was the most likely explanation for the modestly diminished EPEC growth yield in the presence of zinc.

\section{Discussion}

In this report we begin to elucidate the molecular mechanisms by which zinc diminishes EPEC virulence. Though previous data had indicated that zinc reduces LEE gene expression, in a Ler-dependent manner [11], as a negative control in this report we also observed that zinc reduced expression of the bla gene, encoding $\beta$-lactamase. Thus zinc appeared to be a general down regulator of a subset of $E$. coli genes [36], including those associated with EPEC virulence [11,15] (Figure 2). Consistent with this conclusion, we found no evidence for specific regulation by zinc interacting with Ler, or involvement of the major zinc homeostasis regulators Zur or ZntR. However, toward the goal of using dietary supplements to diminish the severity of disease caused by EPEC, and the related EHEC, zinc clearly reduces the expression of BFP, LEE genes, including the LEE1 operon encoding Ler, and stx encoding the Shiga toxin $[11,15]$ (Figure 2).

Looking for a general stress pathway to explain the observed down regulation of EPEC virulence genes, we

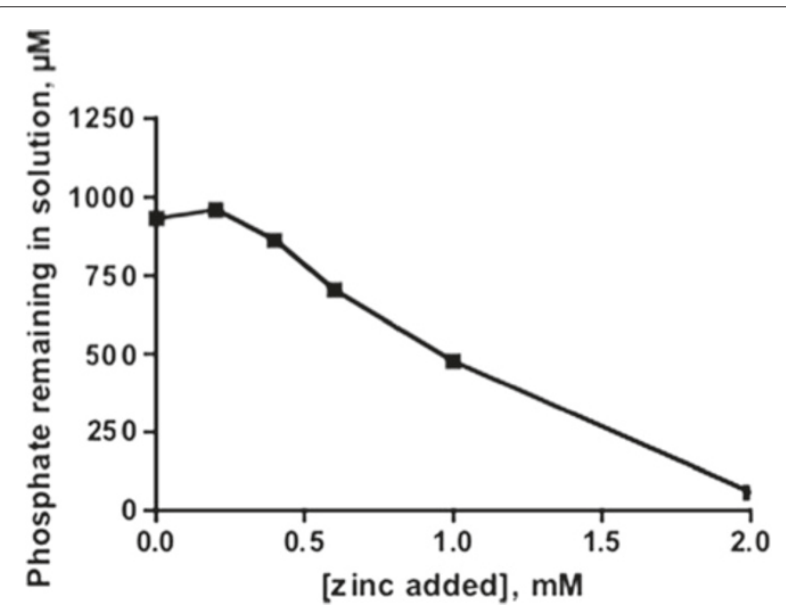

Figure 6 Effect of added zinc on soluble phosphate remaining in DMEM. Zinc acetate was added to DMEM and incubated at $37^{\circ} \mathrm{C}$. Remaining soluble phosphate was quantitated with a Mol-Bio Green assay described in Methods. observed stimulation of rpoE expression in the presence of zinc (Figure 3). We concluded that zinc caused envelope stress to EPEC grown in defined DMEM. Consistent with our observation, rpoE and a number of $r p o E$-dependent genes including $r p o H$ and $h t r A$ were stimulated in the $E$. coli $\mathrm{K}-12$ strain W3110 grown in LB in the presence of zinc chloride [31]. However, it is not likely that the RpoE sigma factor controls expression of LEE genes because the promoters identified for the LEE operons of EPEC were clearly RpoD-dependent, having consensus sequences highly similar to those of promoters transcribed by the $\sigma^{70}$-containing RNA polymerase holoenzyme [14].

Zinc causes envelope stress, in part, by compromising protein tertiary structure, complexing with the thiol side chain of cysteine residues and/or disrupting disulfide bonds. Predictably, extracellular zinc causes a transient induction of the genes necessary for cysteine biosynthesis, thought to mop up excess cytoplasmic zinc [31]. A brief, transitory increase in intracellular zinc concentration most likely occurs inside of the bacterium, particularly for the strains containing mutations in either $z u r$ or $z n t R$, upon addition of 0.3 to $0.5 \mathrm{mM}$ zinc acetate to the culture medium. However, evidence suggests that zinc is quickly complexed to cysteine because the cysteine biosynthetic genes are stimulated by zinc stress [31] and then intracellular zinc concentrations return to normal conditions where free zinc is in the femtomolar range, less than one zinc molecule per bacterium [18].

In EPEC, the type III secretion system is assembled through the envelope, spanning the inner and outer membranes, and beyond, in order to inject effector proteins into the host cell cytoplasm $[12,37,38]$. Thus one would predict that zinc adversely affects the assembly, and integrity of the injectosome once assembled, ultimately preventing protein secretion. Here we demonstrate that zinc physically alters the EPEC envelope (Figure 4) and that the envelope stressor $\mathrm{NH}_{4} \mathrm{VO}_{3}$, which modifies lipid A of the LPS [34] and specifically stimulates the RpoE regulon, inhibits type III protein secretion in a manner similar to that observed for zinc [11] (Figure 5). Thus diminished LEE gene expression and disruption of type III system-dependent protein secretion via envelope stress most likely, largely explain reduced net secretory diarrhoea in the presence of zinc in an ileal loop model of infection using rabbit enteropathogenic $E$. coli $[11,15]$.

The chemical environment within the ileal loops is likely to be altered by the presence of zinc. Notably, our results using the tissue culture medium DMEM (Figure 6) suggest that millimolar quantities of zinc within ileal loops will lead to the precipitation of zinc phosphate and thus reduced availability of phosphate, limiting the number of bacteria within the loops. Zinc acetate levels within the rabbit intestine reached 0.3 to $0.4 \mathrm{mM}$ three days post administering of $10 \mathrm{mg}$ of dietary zinc [15]. Thus this level 
of zinc within the rabbit intestine not only reduces virulence functions of the bacterium, but will also diminish the availability of phosphate. E. coli has two major inorganic phosphate transporters: the Pit system is a high velocity, low affinity system with a $\mathrm{Km}$ of $38.2 \mu \mathrm{M}$, while the Pst system is a low velocity, high-affinity system having a $\mathrm{Km}$ of $0.4 \mu \mathrm{M}$ [39-41]. Therefore, in our experimentation (Figure 6), the level of phosphate did not reach levels low enough to inhibit growth, or reduce the doubling time, even in the presence of $1 \mathrm{mM}$ zinc acetate, but some loss of the overall availability of phosphate in the DMEM resulted in the observed reduced growth yield.

\section{Conclusions}

Zinc interacts with multiple entities in order to affect EPEC virulence- the host, the bacterium itself and the surrounding medium. In humans inadequate levels of dietary zinc lead to an imbalance of the Th1 and Th2 adaptive immune responses, in part by a loss in function of the zinc-containing, thymic hormone thymulin, necessary for T-cell maturation [42]. So certainly, malnourished children in developing countries experiencing zinc deficiencies will have impaired immune function. Previous reports clearly indicate that zinc reduces net secretory diarrhoea in a rabbit ileal loop model of infection $[11,15]$, and our our data now establish that envelope stress and the resultant loss of type III secretion system function begin to explain results observed in the animal infection model. Furthermore, because zinc can be given in relatively large doses without toxicity, this metal ion might also act to remove phosphate from the intestinal lumen, limiting bacterial populations. In sum, our results argue for a more widespread use of dietary zinc supplements to reduce EPEC diarrhoea in children living in the developing regions of the world, but this therapy approach might also be effective against a number of related, type III secretion system containing Gramnegative, diarrhoeal pathogens, for which therapy options are becoming increasingly limited.

\section{Methods}

\section{Bacterial strains and cultures}

The bacterial strains used are listed in Table 1. Overnight cultures were grown at $37^{\circ} \mathrm{C}$ with aeration supplemented with the appropriate antibiotic at the following concentrations: kanamycin $(50 \mu \mathrm{g} / \mathrm{ml})$, ampicillin $(100 \mu \mathrm{g} / \mathrm{ml})$, chloramphenicol $(30 \mu \mathrm{g} / \mathrm{ml})$, or streptomycin $(50 \mu \mathrm{g} / \mathrm{ml})$. Overnight cultures were subcultured into Dulbecco's modified Eagle medium (DMEM) at the dilutions indicated. DMEM in this report refers to DMEM-F12 (SigmaAldrich) containing L-glutamine and $4500 \mathrm{mg} / \mathrm{L}$ glucose, supplemented with $18 \mathrm{mM} \mathrm{NaHCO}_{3}$ and $25 \mathrm{mM}$ HEPES, pH 7.4. DMEM-F12 from Sigma-Aldrich was previously determined to contain no more than $1.5 \mu \mathrm{M}$ zinc [15]. The heavy metal content of the HEPES used in all culture media was measured by the manufacturer (Promega) as less than $5 \mathrm{ppm}$. Therefore the media used in this study contain a negligible amount of zinc compared to the amounts added as a zinc acetate supplement (100 $\mu \mathrm{M}$ or more).

\section{Electrophoretic Mobility Shift Assay (EMSA)}

The LEE4 regulatory fragment (bases -468 to +460 relative to the transription start point) was amplified with primers K1150 and K1153 (Table 2) by PCR using plasmid pJLM165 as template [14]. DNA fragments were separated by $1.0 \%$ agarose gel electrophoresis, stained with ethidium bromide, excised and purified using a QIAQuick Gel Extraction kit (Qiagen). Ler protein was expressed from a pBadMycHis vector and purified as described previously [17]. EMSA-based competition to assess Ler binding to LEE4 regulatory DNA was performed by using nondenaturing $5 \%$ polyacrylamide gels. Polyacrylamide gels were prepared with a 37.5: 1 acrylamide/bisacrylamide solution (Bio-Rad) following a standard protocol. Binding reaction mixtures containing $100 \mathrm{ng}$ DNA, EMSA buffer (10 mM Tris, pH 7.4, $5 \mathrm{mM} \mathrm{NaCl,} 50 \mathrm{mM} \mathrm{KCl,} 50 \mathrm{mg} / \mathrm{ml}$ BSA), $0.5 \mu \mathrm{M}$ Ler, and zinc acetate at the indicated concentrations were incubated at room temperature for 15 min. After the addition of glycerol to a concentration of $2.5 \%(\mathrm{v} / \mathrm{v})$, samples were separated by electrophoresis at $4^{\circ} \mathrm{C}$ overnight at $35 \mathrm{~V}$. Gels were stained with ethidium bromide and imaged using a Bio-Rad Fluor-S Multilmager. Band intensities were quantified with the Gnu Image Manipulation Program (http://www.gimp.org/).

\section{$\boldsymbol{\beta}$-galactosidase assays}

Plasmid pJLM164 containing a LEE1-lacZ fusion, and plasmid pJLM165 containing a LEE4-lacZ fusion were transformed into EPEC strains E2348/69 and LRT9, and into the plasmid-cured EPEC derivative JPN15 and the K12 strain MC4100. Strains were cultured overnight in LB medium with $50 \mu \mathrm{g} / \mathrm{ml}$ kanamycin and then subcultured 1:100 into $3 \mathrm{ml}$ DMEM buffered with $25 \mathrm{mM}$ HEPES, $\mathrm{pH} 7.4$, in the presence and absence of $0.3-0.5 \mathrm{mM}$ zinc acetate. Cells were harvested with $\mathrm{OD}_{600}$ between 0.3 and 0.5 , and $\beta$-galactosidase activity was monitored by standard methods [32]. Three independent assays were performed from each culture.

\section{Disk diffusion assays}

MC4100-derived strains JLM164, JLM165, MCamp, and CT32 containing single-copy lacZ reporter fusions were plated evenly on DMEM agar containing $30 \mu \mathrm{g} / \mathrm{ml} \mathrm{X-gal.}$ Sterile disks containing $15 \mu \mathrm{l}$ of varying concentrations of zinc acetate were placed on the plates which were then cultured overnight at $37^{\circ} \mathrm{C}$. The plates were then moved to $4^{\circ} \mathrm{C}$ for 6 hours to develop the color and photographed. 
Table 2 Oligonucleotide primers used in this study

\begin{tabular}{lrccc}
\hline Primer & Sequence $\mathbf{5}^{\prime} \mathbf{-} \mathbf{3}^{\prime}$ & Strand & Target & Reference \\
\hline K1153 & CCGGAATTCTGCCGATGGCACCAGACA & + & LEE4 & [14] \\
K1150 & CGCGGATCCTGCCAAACATCGCCAAAGTAG & - & LEE4 & {$[14]$} \\
\hline
\end{tabular}

\section{Transmission electron microscopy}

Overnight cultures of EPEC bacteria grown in LB medium were diluted 1:100 into DMEM-F12 medium, described above. After 10 hours of incubation at $37^{\circ} \mathrm{C}$ with shaking, incubation was continued in the absence and presence of final concentration $0.3 \mathrm{mM}$ zinc acetate. After 15 hours of growth, samples were pelleted by centrifugation, spent medium discarded, and resuspended in $100 \mu \mathrm{l} 0.1 \mathrm{M}$ $\mathrm{MgSO}_{4}$. Forty $\mu \mathrm{l}$ samples were pipetted onto parafilm, and carbon-formvar grids, shiny side down, were placed on top of each drop and left for 7 minutes. The grid was then transferred to a $40-\mu$ l drop of $1.3 \%$ uranyl acetate in water and left to stain for 40 seconds. The grid was then removed and blotted dry. Samples were viewed on a Philips CM120 transmission electron microscope. Negatively stained samples were viewed at ambient temperature. Images were recorded at 16-bit grayscale in Gatan digital micrograph 3 (DM3) format on a $1,024 \times 1,024$ pixel Gatan 794 charge-coupled device multiscan camera. Gatan digital micrograph 3.4.0 software was used to calculate power spectra and to convert DM3 format raw images and power spectra to eight-bit grayscale TIF images. Grids were imaged at the Oregon Health \& Science University Electron Microscopy Core Facility.

\section{Secretion assays}

EPEC strain E2348/69 was cultured overnight in $2 \mathrm{ml} \mathrm{LB}$ medium; the EPEC escN deletion strain CVD452 was similarly grown in LB containing $50 \mu \mathrm{g} / \mathrm{ml}$ kanamycin. The cultures were diluted 100-fold into $20 \mathrm{ml}$ DMEM containing 25 mM HEPES, pH 7.4, and various concentrations of zinc acetate or ammonium metavanadate in 125 ml flasks. Ammonium metavanadate was added from a $0.1 \mathrm{~N}$ stock solution prepared by adding the appropriate amount of solid $\mathrm{NH}_{4} \mathrm{VO}_{3}$ and raising the $\mathrm{pH}$ until complete dissolution was achieved; tests showed that adding $\mathrm{NH}_{4} \mathrm{VO}_{3}$ from this stock up to $2 \mathrm{mM}$ did not change the $\mathrm{pH}$ of DMEM solutions appreciably. The cultures were grown statically overnight at $37^{\circ} \mathrm{C}$, reaching an $\mathrm{OD}_{600}$ of $0.9-1.1$. Cells were removed from the medium by centrifugation at $12,000 \mathrm{x}$ g for 30 minutes. Secreted proteins remaining in the supernatant were precipitated by adding $25 \%$ trichloroacetic acid to a volume of supernatant chosen such that volume $(\mathrm{ml}) \times$ culture $\mathrm{OD}_{600}=6.0$. Precipitated proteins in this volume were pelleted by multiple spins into the same $1.5 \mathrm{ml}$ collection tube at $12,000 \mathrm{~g}$. Pellets were washed with acetone, dried at $95^{\circ} \mathrm{C}$ for 10 minutes, then resuspended by heating with Laemmli sample buffer (0.1 M Tris-Cl pH 6.8, 5\% glycerol, $2 \% \beta$-mercaptoethanol, $2 \%$ sodium docedcyl sulfate, $0.01 \%$ bromophenol blue) at $95^{\circ} \mathrm{C}$ for 10 minutes. Proteins were separated on $15 \%$ SDS-polyacrylamide gels and visualized by Coomassie blue staining. To verify the identity of the secreted protein, the band migrating at $33 \mathrm{kDa}$ was cut out of the gel, and an in-gel tryptic digest was performed according to the manufacturer's instructions (Promega; mass spectrometry grade Trypsin and Protease Max surfactant). MALDI-TOF mass spectrometry was used to confirm the presence of EspB tryptic fragments in the digest.

\section{Determination of phosphate in DMEM containing Zinc}

DMEM was supplemented with $0-2.0 \mathrm{mM}$ zinc acetate and allowed to incubate at $37^{\circ} \mathrm{C}$ for 2 hours. Insoluble material was removed by spin filtration, and then soluble phosphate was quantitated using a Bio-Mol Green assay (Enzo Life Sciences).

\section{Competing interests}

The authors declare that they have no competing interests.

\section{Authors' contributions}

Experiments were performed by the following authors: EMSA assays - GCB and MT; Miller assays - GCB, JLM, KT, MT, and NRE; disc assays for gene expression and growth inhibition - MT and NRE; secretion assays - GCB; zinc precipitation measurements - JC; transmission electron microscopy - NRE. The manuscript was written primarily by JLM with review by all authors before submission. All authors read and approved the final manuscript.

\section{Acknowledgements}

We gratefully acknowledge Dr. Eric Barklis, Director of the OHSU EM Core Facility, and Jake Eccles for imaging samples by TEM. This work was supported by $\mathrm{NIH}$ grant 5R01Al081528-02 awarded to J. Crane, E. Boedeker, and J. Mellies. Identification of the secreted protein EspB by mass spectrometry was supported, in part, by Award Number P30ES000210 from the National Institute of Environmental Health Sciences (NIEHS), National Institutes of Health (NIH). The content is solely the responsibility of the authors and does not necessarily represent the official views of NIEHS or NIH. The authors acknowledge the Biomolecular Mass Spectrometry Core of the Environmental Health Sciences Core Center at Oregon State University.

\section{Author details}

1 Biology Department, Reed College, Portland OR, USA. ${ }^{2}$ Division of Infectious Diseases, University at Buffalo, Buffalo, New York, USA. ${ }^{3}$ Division of

Gastroenterology, University of New Mexico, and the Albuquerque VA Medical Center, Albuquerque, New Mexico, USA.

Received: 11 January 2012 Accepted: 12 June 2012

Published: 24 June 2012

\section{References}

1. Abba K, Sinfield R, Hart C, Garner P: Pathogens associated with persistent diarrhoea in children in low and middle income countries: systematic review. BMC Infect Dis 2009, 9:88. 
2. Kaper J, Nataro J, Mobley H: Pathogenic Escherichia coli. Nat Rev Microbiol 2004, 2:123-140.

3. Clarke S, Haigh R, Freestone P, Williams P: Virulence of enteropathogenic Escherichia coli, a global pathogen. Clin Microbiol Rev 2003, 16:365-378

4. Lim J, Yoon J, Hovde C: A brief overview of Escherichia coli 0157:H7 and its plasmid 0157. J Microbiol Biotechnol 2010, 20:5-14.

5. Sazawal S, Black R, Bhan M, Bhandari N, Sinha A, Jalla S: Zinc supplementation in young children with acute diarrhea in India. $N$ Engl J Med 1995, 333:839-844.

6. Yakoob M, Theodoratou E, Jabeen A, Imdad A, Eisele T, Ferguson J, Jhass A, Rudan I, Campbell H, Black R, Bhutta Z: Preventive zinc supplementation in developing countries: impact on mortality and morbidity due to diarrhea, pneumonia and malaria. BMC Public Health 2011, 11(Suppl 3):S23.

7. McCall K, Huang C, Fierke C: Function and mechanism of zinc metalloenzymes. J Nutr 2000, 130:1437S-1446S.

8. Overbeck S, Rink L, Haase H: Modulating the immune response by oral zinc supplementation: a single approach for multiple diseases. Arch Immunol Ther Exp (Warsz) 2008, 56:15-30.

9. Prasad A: Impact of the discovery of human zinc deficiency on health. J Am Coll Nutr 2009, 28:257-265.

10. Crane J, Hoque $K$ : Zinc for infectious diarrhea in developed countries: should we be sprinkling our own lawns?. J Pediatr Gastroenterol Nutr 2008, 46:484-485.

11. Crane J, Naeher T, Shulgina I, Zhu C, Boedeker E: Effect of zinc in enteropathogenic Escherichia coli infection. Infect Immun 2007 75:5974-5984.

12. Cornelis G: The type III secretion injectisome. Nat Rev Microbio/ 2006, 4:811-825.

13. Elliott S, Wainwright L, McDaniel T, Jarvis K, Deng Y, Lai L, McNamara B, Donnenberg M, Kaper J: The complete sequence of the locus of enterocyte effacement (LEE) from enteropathogenic Escherichia coli e2348/69. Mol Microbiol 1998, 28:1-4.

14. Mellies J, Elliott S, Sperandio V, Donnenberg M, Kaper J: The Per regulon of enteropathogenic Escherichia coli: identification of a regulatory cascade and a novel transcriptional activator, the locus of enterocyte effacement (LEE)-encoded regulator (Ler). Mol Microbiol 1999, 33:296-306.

15. Crane J, Byrd I, Boedeker E: Virulence inhibition by zinc in shiga-toxigenic Escherichia coli. Infect Immun 2011, 79:1696-1705.

16. Torres A, López-Sánchez G, Milflores-Flores L, Patel S, Rojas-López M, Martínez de la Peña C, Arenas-Hernández M, Martínez-Laguna Y: Ler and $\mathrm{H}-\mathrm{NS}$, regulators controlling expression of the long polar fimbriae of Escherichia coli 0157:H7. J Bacteriol 2007, 189:5916-5928.

17. Mellies J, Benison G, McNitt W, Mavor D, Boniface C, Larabee F: Ler of pathogenic Escherichia coli forms toroidal protein-DNA complexes. Microbiology 2011, 157:1123-1133.

18. Outten C, O'Halloran T: Femtomolar sensitivity of metalloregulatory proteins controlling zinc homeostasis. Sci 2001, 292:2488-2492.

19. Levine M: Escherichia coli that cause diarrhea: enterotoxigenic, enteropathogenic, enteroinvasive, enterohemorrhagic, and enteroadherent. J Infect Dis 1987, 155:377-389.

20. Jerse A, Yu J, Tall B, Kaper J: A genetic locus of enteropathogenic Escherichia coli necessary for the production of attaching and effacing lesions on tissue culture cells. Proc Natl Acad Sci U SA 1990, 87:7839-7843.

21. Casadaban M: Transposition and fusion of the lac genes to selected promoters in Escherichia coli using bacteriophage lambda and Mu. J Mol Biol 1976, 104:541-555.

22. Patzer $S$, Hantke $K$ : The zinc-responsive regulator Zur and its control of the znu gene cluster encoding the ZnuABC zinc uptake system in Escherichia coli. J Biol Chem 2000, 275:24321-24332.

23. Pruteanu M, Neher S, Baker T: Ligand-controlled proteolysis of the Escherichia coli transcriptional regulator ZntR. J Bacterio/ 2007, 189:3017-3025

24. Tam C, Missiakas D: Changes in lipopolysaccharide structure induce the sigma(E)-dependent response of Escherichia coli. Mol Microbiol 2005, 55:1403-1412.

25. Sperandio V, Mellies J, Nguyen W, Shin S, Kaper J: Quorum sensing controls expression of the type III secretion gene transcription and protein secretion in enterohemorrhagic and enteropathogenic Escherichia coli. Proc Natl Acad Sci U S A 1999, 96:15196-15201.

26. Jarvis K, Girón J, Jerse A, McDaniel T, Donnenberg M, Kaper J: Enteropathogenic Escherichia coli contains a putative type III secretion system necessary for the export of proteins involved in attaching and effacing lesion formation. Proc Natl Acad Sci U S A 1995 92:7996-8000.

27. Ferreira G, Spira B: The pst operon of enteropathogenic Escherichia coli enhances bacterial adherence to epithelial cells. Microbiology 2008, 154:2025-2036.

28. Simons R, Houman F, Kleckner N: Improved single and multicopy lac-based cloning vectors for protein and operon fusions. Gene 1987, 53:85-96.

29. Outten C, Outten F, O'Halloran T: DNA distortion mechanism for transcriptional activation by ZntR, a Zn(II)-responsive MerR homologue in Escherichia coli. J Biol Chem 1999, 274:37517-37524.

30. Egler M, Große C, Grass G, Nies D: Role of the extracytoplasmic function protein family sigma factor RpoE in metal resistance of Escherichia coli. J Bacteriol 2005, 187:2297-2307.

31. Yamamoto K, Ishihama A: Transcriptional response of Escherichia coli to external zinc. J Bacteriol 2005, 187:6333-6340.

32. Miller JH: Experiments in Molecular Genetics. Cold Spring Harbor, New York: Cold Spring Harbor Press; 1972.

33. Ades S: Regulation by destruction: design of the $\sigma^{\mathrm{E}}$ envelope stress response. Curr Opin Microbiol 2008, 11:535-540.

34. Zhou Z, Lin S, Cotter R, Raetz C: Lipid A modifications characteristic of Salmonella typhimurium are induced by $\mathrm{NH}_{4} \mathrm{VO}_{3}$ in Escherichia coli K12: detection of 4-amino-4-deoxy-L-arabinose, phosphoethanolamine and palmitate. J Biol Chem 1999, 274:18503-18514.

35. Mellies J, Haack K, Galligan D: SOS regulation of the type III secretion system of enteropathogenic Escherichia coli. J Bacteriol 2007, 189:2863-2872

36. Lee L, Barrett J, Poole R: Genome-wide transcriptional response of chemostat-cultured Escherichia coli to zinc. J Bacterio/ 2005, 187:1124-1134

37. Galán J, Wolf-Watz H: Protein delivery into eukaryotic cells by type III secretion machines. Nature 2006, 444:567-573.

38. Diepold A, Amstutz M, Abel S, Sorg I, Jenal U, Cornelis G: Deciphering the assembly of the Yersinia type III secretion injectisome. EMBO 2010, 29:1928-1940

39. Willsky G, Malamy M: Control of the synthesis of alkaline phosphatase and the phosphate-binding protein in Escherichia coli. J Bacteriol 1976, 127:595-609.

40. Willsky G, Malamy M: Characterization of two genetically separable inorganic phosphate transport systems in Escherichia coli. J Bacteriol 1980, 144:356-365.

41. Wanner B: Chapter 87: Phosphorus Assimilation and Control of the Phosphate Regulon. [http://ecosal.org].

42. Prasad A: Zinc: mechanisms of host defense. J Nutr 2007, 137:1345-1349.

doi:10.1186/1471-2180-12-123

Cite this article as: Mellies et al:: Zinc-induced envelope stress diminishes type III secretion in enteropathogenic Escherichia coli. BMC Microbiology 2012 12:123. 